\title{
Research on Hyper pipes and Voting Feature Intervals Classifier for Condition Monitoring of Wind Turbine Blades Using Vibration Signals
}

\author{
A. Joshuva, S. Sivakumar, R. Vishnuvardhan, \\ G. Deenadayalan, R. Sathishkumar
}

\begin{abstract}
Renewable energy is viewed as a vital energy field due to the present energy devastations. Among the vital substitutions being considered, wind energy is a strong challenger as a result of its reliability. "To yield wind energy more effectively, the structure of wind turbines has designed bigger, making protection and restoration works difficult. Because of different natural conditions, wind turbine blades are exposed to vibration and it prompts failure. If the failure is not analyzed initially, then it will haste dreadful destruction of the turbine structure. To increase safety perceptions, to decrease down time and to cut down the repeat of unpredictable breakdowns, the wind turbine blades must be examined from time to time to guarantee that they are in great condition. In this paper, a three bladed wind turbine was preferred and using vibration source through statistical features, the state of a wind turbine blade is inspected. The fault classification is carried out using machine learning techniques like hyperpipes (HP) and voting feature intervals (VFI) algorithm. The performance of these algorithms is compared and better algorithm is suggested for fault prediction on wind turbine blades."
\end{abstract}

Keywords--- Condition Monitoring, Fault Diagnosis, Voting Feature Interval Algorithm, Hyperpipes, Statistical Features, Vibration Signals.

\section{INTRODUCTION}

Wind energy has become the most needed resource in the present period because of overall environmental pollute [1] Wind energy is one of the proficient renewable sources and option for normally used sources. The wind turbine is used to change over wind energy into electrical energy. To make wind energy more important assets of energy, performance, security, dependability, feasibility and the life of turbines must be upgraded [2]. The wind makes the rotor post turn, in this way, making the pole inside the wind turbine turn. The fragile, long and flexible, sharp edge blades are the part sticks through the most incredibly complex force and it is easy to bring the vibration on sharp edge blades [3]. The stressed and dangerous vibration will incite pulverization to the rotor edge and cause damage. The blade is one of the crucial parts with frequent damages. Because of its massive advancement and working condition, the vibration of the

Manuscript received September 16, 2019.

A. Joshuva*, Department of Mechanical Engineering, Hindustan Institute of Technology and Science, Chennai, T.N, India.

S. Sivakumar, Department of Mechanical Engineering, Hindustan Institute of Technology and Science, Chennai, T.N, India.

R. Vishnuvardhan, Department of Mechatronics Engineering, Sri Krishna College of Engineering and Technology, Coimbatore. T.N, India.

G. Deenadayalan, Department of Mechanical Engineering, Hindustan Institute of Technology and Science, Chennai, T.N, India.R. Sathishkumar, Department of Automobile Engineering, Hindustan Institute of Technology and Science, Chennai, T.N, India. blade is hard to figure it while working. In this study, damage identification on wind turbine blades is carried out using vibration source and machine learning approach [4].

Much research and study were carried out on failure analysis of the blade. To name a few, Simulation of fatigue failure in a full composite wind turbine blade was studied by Mahmood M. Shokrieh, RohamRafiee [5]. The lifetime prediction of a horizontal axis wind turbine composite blade is considered. Load cases are identified, calculated and evaluated. Static analysis is performed with a full 3-D finite element method.

They predicted 21 years approximately using ANSYS. Andrew Kusiak and Anoop Verma [6] carried out work on a data-driven approach for monitoring blade pitch faults in wind turbines using SCADA data. They considered two blade pitch faults namely, blade angle asymmetry and blade angle implausibility and determine the associations between them.

Frost et. al[7] carried out a study on integrating structural health management with contingency control for wind turbines using nonlinear high-fidelity simulation. The structural health and contingency control of the blade was studied.

The speed of the turbine and decision making using prognostic information was also carried out.Bindi Chen et. al[8] conducted an experiment on wind turbine pitch faults prognosis using a-priori knowledge based adaptive neurofuzzy inference system (ANFIS) using SCADA data and obtained $88.30 \%$ classification accuracy.

Lee et. al[9] done a work on wind turbine blade moment signals to blade condition monitoring using a transformation algorithm.

They presented a novel method of transforming blade moment signals on a horizontal axis 3-blade wind turbine. Simulation of aero-elastic behavior in a composite wind turbine blade was studied by Rafieeet. al[10]. Aero-elastic analysis of a full scale composite wind turbine blade was investigated using 3D model and aerodynamic loading was determined using modified Blade Element Momentum (BEM) theory and Computational Fluid Dynamics (CFD) method.

Bessa et. al[11] carried out a work on data driven fault detection and isolation scheme for a wind turbine benchmark.

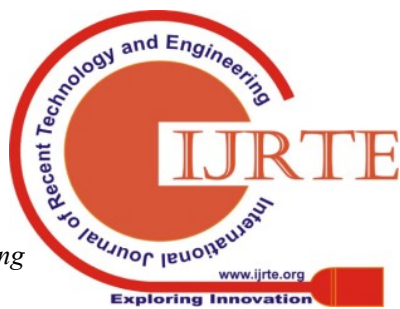


In their first step, the fault detection, is based on an alternative method based on the Gibbs sampling algorithm in which the occurrence of a sensor fault is modelled as a change point detection in a time series.

On the second step, the fault isolation, is handled via Fuzzy/Bayesian network scheme classifying the kind of fault. Optimal selection of auto regressive model coefficients for early damage detects ability with an application to wind turbine blades was carried out by Hoell and Omenzetter [12].

This study enhances the selection of auto regressive model coefficients for statistical hypothesis testing for damage presence and adding or eliminating the coefficients is carried out by genetic algorithm.

Aero-structural design and optimization of a small wind turbine blade study was carried out by Pourrajabian et. al[13].

They developed a methodology for aero-structural design, including consideration of the starting of a small wind turbine blade. They carried out both structural analysis and stress analysis on the blade by optimization. Damir et. al [14] has done a numerical model for robust shape optimization of wind turbine blades using 3D geometric modeler. They considered the blade design using simulation process, but they didn't focus on the faults which affects the performance of the wind turbine. Numerous works were carried out using simulation analysis of fault and design analysis of wind turbine blade; however, only a very few in the experimental analysis was carried out [15].

The machine learning technique was considered for wind turbine blade fault diagnosis; however, the usage was limited in the literature.

A very limited set of defects was considered for analysis. This is especially true in the case of fault diagnosis of wind turbine blade.

Hence, there is a strong need to design a fault diagnosis system which can handle multiple faults in wind turbine blades using machine learning approaches. This study makes a novel attempt to find different blade faults applying machine learning approach and statistical features. Figure 1 shows the methodology of the work done.

In this study for wind turbine blade fault diagnosis,

- Faults like pitch angle twist, blade bends, erosion, hub-blade loose connection and crack are considered

- Vibration signals are taken for the blade using data acquisition.

- Statistical method is used for feature extraction

- $\quad \mathrm{J} 48$ algorithm is used for feature selection

- $\quad$ Hyperpipes (HP) and voting feature intervals (VFI) algorithm is used for feature classification

- Better classifier is suggested for the real-time assessment

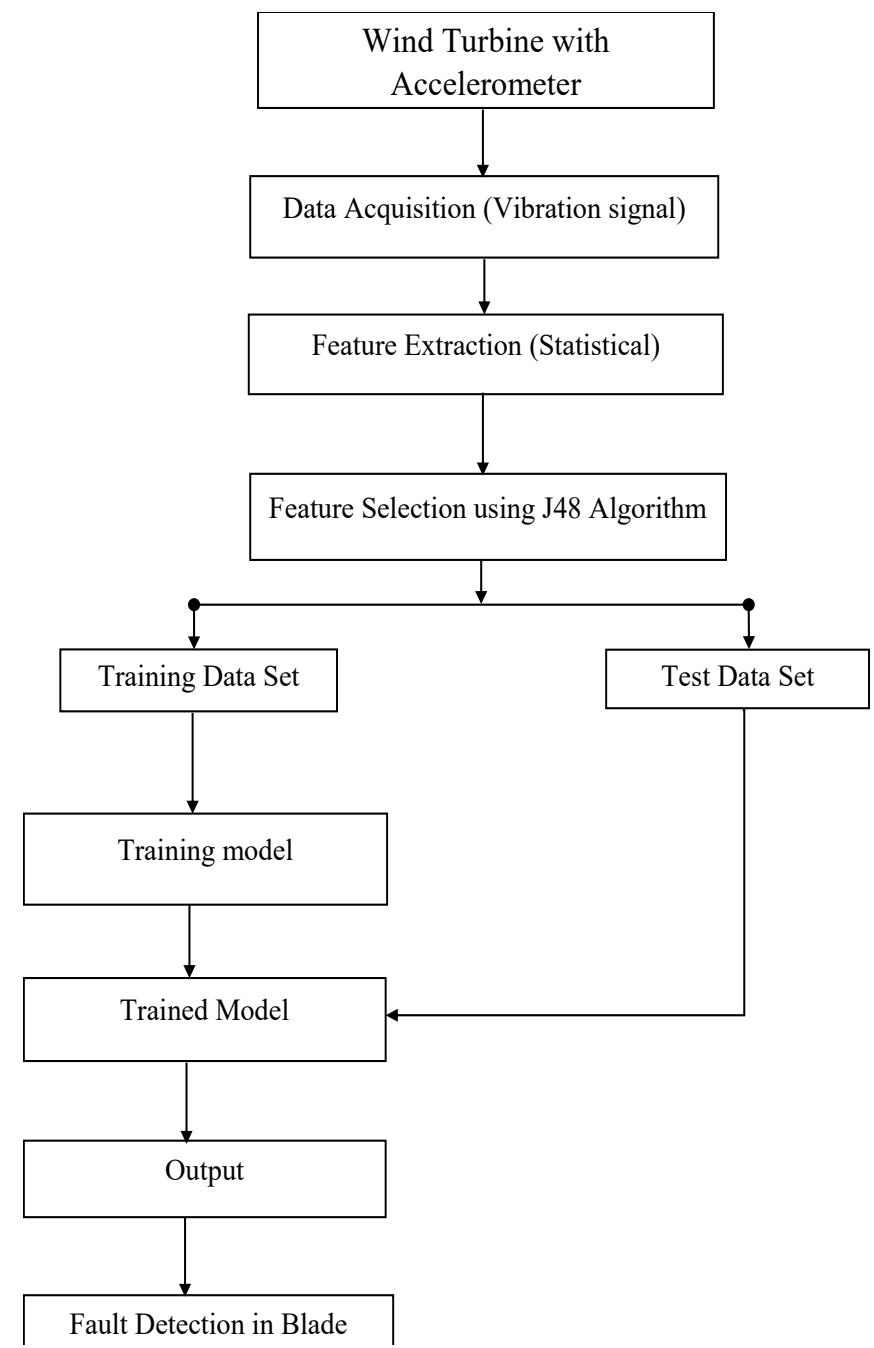

Figure 1: Methodology

\section{EXPERIMENTAL STUDIES}

The main aim of this study is to classify whether the blades are in good condition or in a defective state. If it is defective, then the objective is to identify the type of fault. The experimental setup and experimental procedure are described in the following subsections.

\subsection{Experimental Setup}

The experiment was carried out on a $50 \mathrm{~W}, 12 \mathrm{~V}$ variable speed wind turbine (MX-POWER, model: FP-50W-12V). The technical parameters of a wind turbine are given in Table 1. The wind turbine was mounted on a fixed steel stand in front of the open circuit wind tunnel outlet. The wind tunnel acts as a wind source to start the wind turbine. The wind speed was varied continuously in order to simulate the environmental wind condition. The experimental setup is shown in Figure 2. Piezoelectric type accelerometer was used as a transducer for acquiring vibration signals. It has high sensitivity for detecting faults. Hence, accelerometers are widely used in condition monitoring. In this case, an uniaxial accelerometer of $500 \mathrm{~g}$ range, $100 \mathrm{mV} / \mathrm{g}$ sensitivity, and resonant frequency around $40 \mathrm{~Hz}$ was used. 
Table 1: Technical parameters of wind turbine

\begin{tabular}{|l|l|}
\hline Model & FP-50W-12V \\
\hline Rated Power & $50 \mathrm{~W}$ \\
\hline Rated Voltage & $12 \mathrm{~V}$ \\
\hline Maximum Current & $4 \mathrm{~A}$ \\
\hline $\begin{array}{l}\text { Rated Rotating } \\
\text { Rate }\end{array}$ & $850 \mathrm{rpm}$ \\
\hline $\begin{array}{l}\text { Start- } \\
\text { up Wind Speed }\end{array}$ & $2.5 \mathrm{~m} / \mathrm{s}$ \\
\hline Cut-in Wind Speed & $3.5 \mathrm{~m} / \mathrm{s}$ \\
\hline $\begin{array}{l}\text { Cut- } \\
\text { out Wind Speed }\end{array}$ & $15 \mathrm{~m} / \mathrm{s}$ \\
\hline $\begin{array}{l}\text { Security Wind Spee } \\
\text { d }\end{array}$ & $40 \mathrm{~m} / \mathrm{s}$ \\
\hline Rated Wind Speed & $12.5 \mathrm{~m} / \mathrm{s}$ \\
\hline Engine & $\begin{array}{l}\text { Three- } \\
\text { phase permanent magnet gene } \\
\text { rator }\end{array}$ \\
\hline Rotor Diameter & $1050 \mathrm{~mm}$ \\
\hline Blade Material & $\begin{array}{l}\text { Carbon fiber reinforced plasti } \\
\text { cs }\end{array}$ \\
\hline
\end{tabular}

The piezoelectric accelerometer (DYTRAN 3055B1) was mounted on the nacelle near to the wind turbine hub to record the vibration signals using an adhesive mounting technique. It was connected to the DAQ system through a cable. The data acquisition system (DAQ) used was NI USB 4432 model. The DAQ card has five analogue input channels with a sampling rate of 102.4-kilo samples per second with 24-bit resolution. The accelerometer is coupled to a signal conditioning unit which consists of an inbuilt charge amplifier and an analogue-to-digital converter (ADC). From the ADC, the vibration signal was taken. These vibration signals were used to extract features through feature extraction technique. One end of the cable is plugged to the accelerometer and the other end to the AIO port of DAQ system. NI - LabVIEW was used to interface the transducer signal and the system (PC).

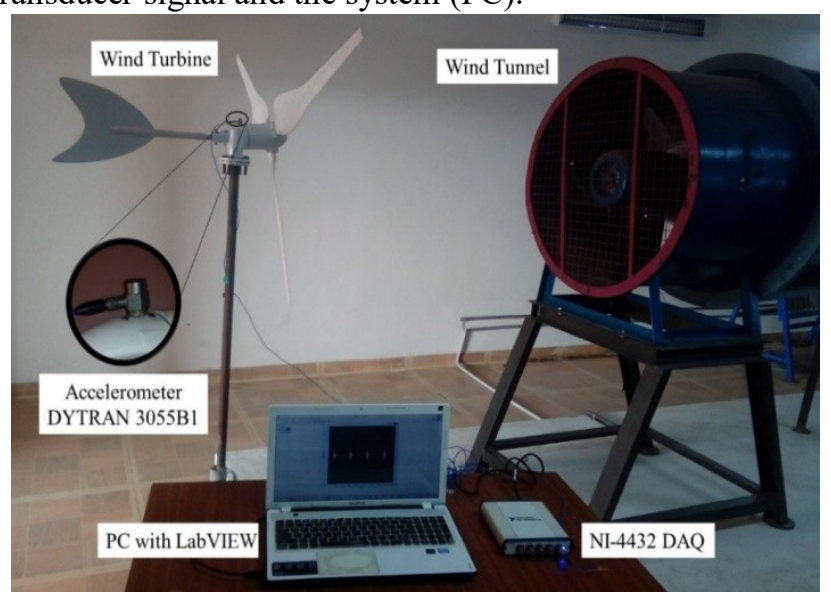

Figure 2: Wind turbine setup

\subsection{Experimental procedure}

In the present study, three-blade variable horizontal axis wind turbine (HAWT) was used. Initially, the wind turbine was considered to be in good condition (free from defects, new setup) and the signals were recorded using an accelerometer. These signals were recorded with the following specifications.
1. Sample length: The sample length was chosen long enough to ensure data consistency; and also the following points were considered. ARMA measures are more meaningful when the number of samples is sufficiently large. On the other hand, as the number of samples increases the computation time increases. To strike a balance, a sample length of 10000 was chosen.

2. Sampling Frequency: The sampling frequency should be at least twice the highest frequency contained in the signal as per Nyquist sampling theorem. By using this theorem sampling frequency was calculated as $12 \mathrm{kHz}(12000 \mathrm{~Hz})$.

3. Number of samples: Minimum of 100 (hundred) samples were taken for each condition of the wind turbine blade and the vibration signals were stored in data files.

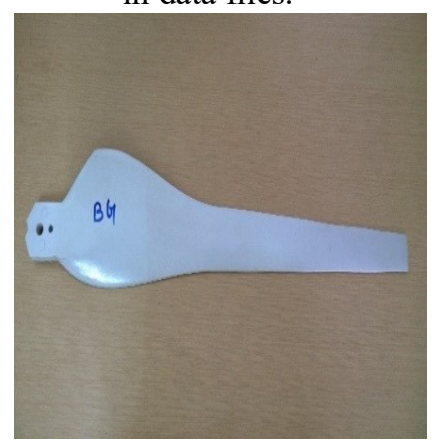

Good condition blade

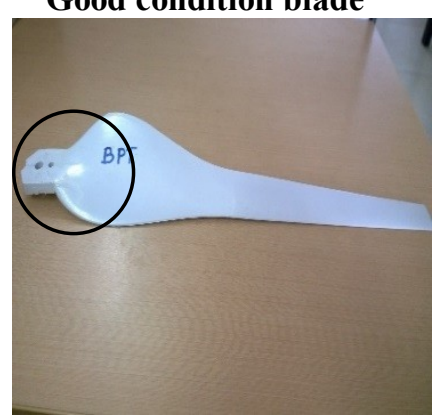

Blade with pitch angle twist

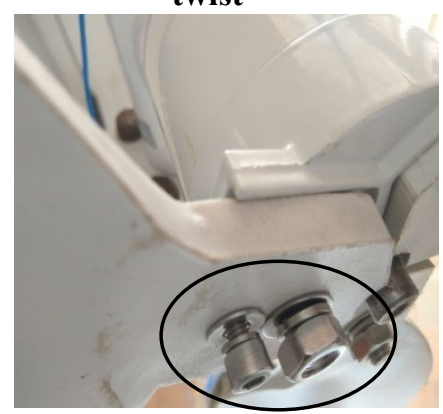

Hub-blade loose connection

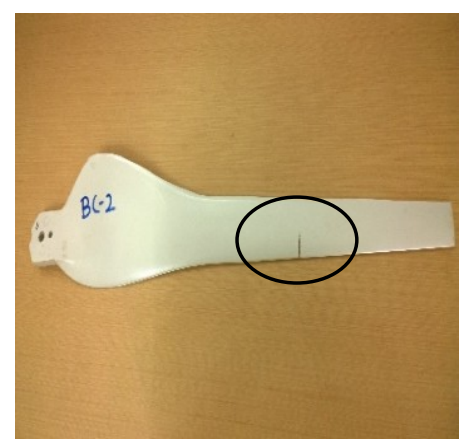

Blade with crack

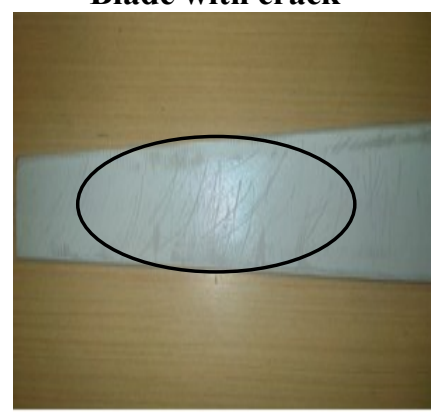

Blade with erosion

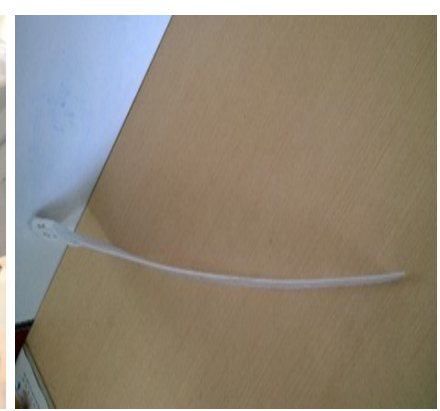

Blade with bend (Top

View)
Figure 3: Various blade fault conditions

The following faults were simulated one at a time while all other components remain in good condition and the corresponding vibration signals were acquired. Figure 3 shows the different blade fault conditions which are simulated on the blade.

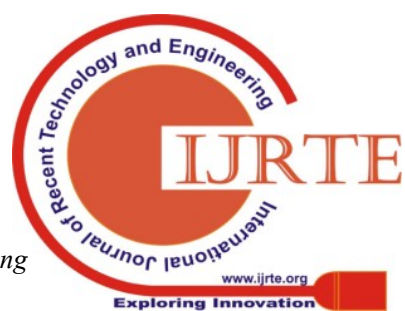


a) Blade bend (BB): This fault occurs due to the highspeed wind and complex forces caused by the wind. The blade was made to flap wise bend with $10^{\circ}$ angle.

b) Blade crack (BC-2): This occurs due to foreign object damage on the blade while it is in operating condition. On the blade, $15 \mathrm{~mm}$ crack was made.
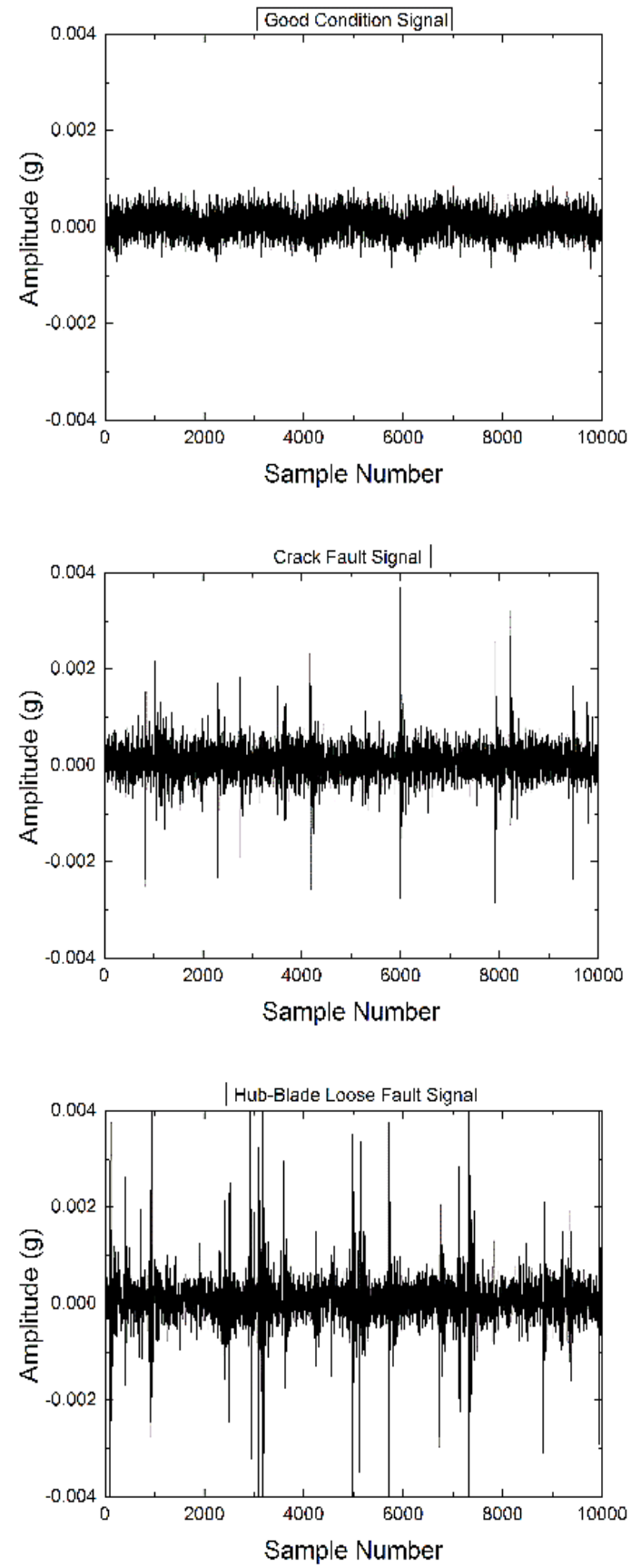

c) Blade erosion (BE): This fault is due to the erosion of the top layer of the blade by the high-speed wind. The smooth surface of the blade was eroded using emery sheet $(320 \mathrm{Cw})$ to provide an erosion effect on the blade.
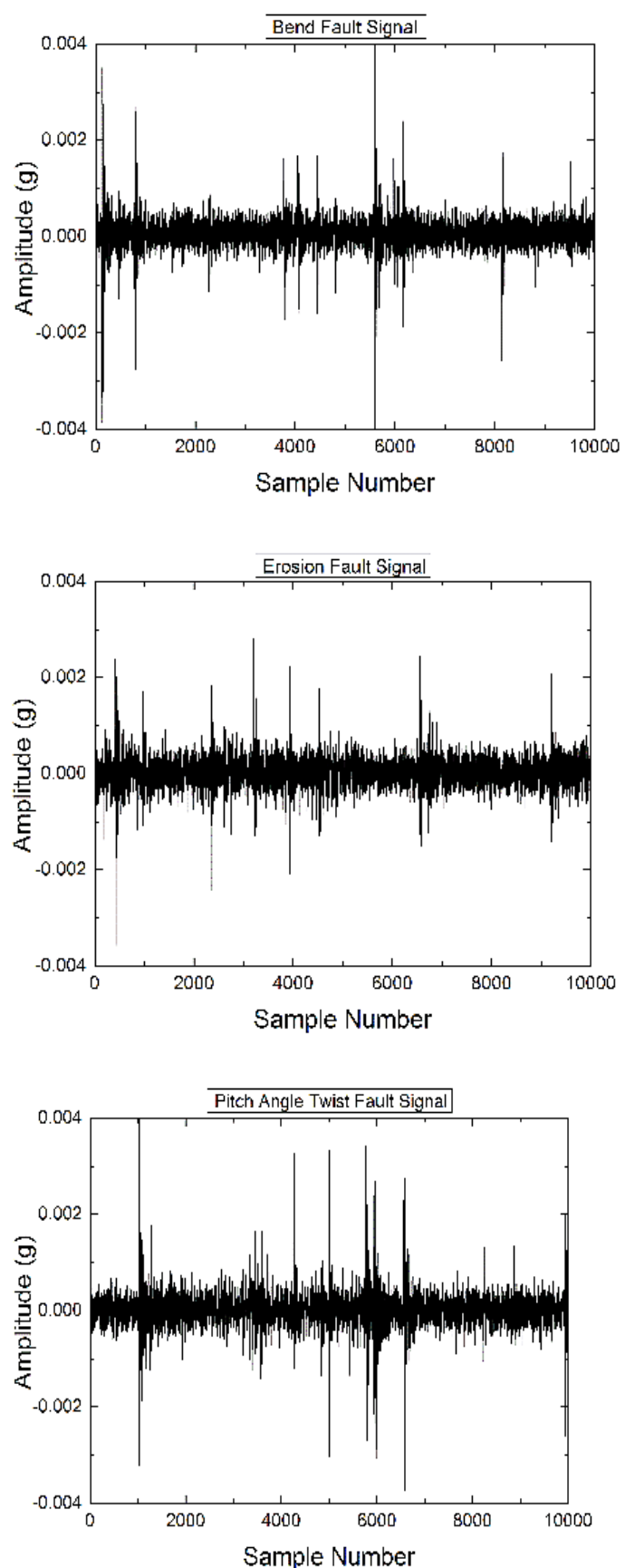

Figure 4: Vibration signal plot

d) Hub-blade loose contact: This fault generally occurs on a wind turbine blade due to an excessive runtime or usage time. The bolt connecting the hub and blade was made loose to obtain this fault.

e) Blade pitch angle twist (PAT): This fault occurs due to the stress on the blade caused by high-speed wind. This makes the pitch get twisted, creating a heavy vibration to the framework. To attain this fault, blade pitch was twisted about $12^{\circ}$ angle with respect to the normal blade condition.
In Figure 4, the vibration signals (sample number vs. amplitude) are shown which were taken for different conditions of the wind turbine blade (good condition blade, blade bend, blade erosion, hub-blade loose connection, blade crack and pitch angle twist). 


\section{STATISTICAL METHOD FOR FEATURE EXTRACTION}

The vibration signals were obtained for good and other faulty conditions of the blades. If the time domain sampled signals are given directly as inputs to a classifier, then the number of samples should be constant. The number of samples obtained are the function of rotation of the blade speed. Hence, it cannot be used directly as the input to the classifier. However, a few features must be extracted before the classification process. Descriptive statistical parameters [16] such as sum, mean, median, mode, minimum, maximum, range, skewness, kurtosis, standard error, standard deviation and sample variance were computed to serve as features in the feature extraction process.

- Sum: It is the sum of all feature values for each sample.

- Mean: The arithmetic average of a set of values or distribution.

- Median: Middle value sorting out the greater and lesser splits of a data set.

- Mode: Most frequent value available in the data set.

- Skewness: Skewness illustrates the degree of irregularity of a distribution around its mean. The following formula was used for calculation of skewness.

$$
\text { Skewness }=\frac{n}{(n-1)(n-2)} \sum\left(\frac{x_{i}-\bar{x}}{\mathrm{~s}_{\mathrm{d}}}\right)^{3}-(1)
$$

- Kurtosis: Kurtosis point toward the flatness or the spikiness of the signal. Its value is very low for normal condition of the blade and high for the faulty condition of the blade due to the spiky nature of the signal and ' $\mathrm{s}$ ' is the sample standard deviation

Kurtosis $=\left\{\frac{n(n+1)}{(n-1)(n-2)(n-3)} \sum\left(\frac{x_{i}-\bar{x}}{\mathrm{~s}_{\mathrm{d}}}\right)^{4}\right\}-\frac{3(n-1)^{2}}{(n-2)(n-3)}$ (2)

- Standard error: Standard error is a measure of the amount of error in the prediction of $y$ for an individual $\mathrm{x}$ in the regression, where $\mathrm{x}$ and $\mathrm{y}$ are the sample means and ' $\mathrm{n}$ ' is the sample size.

$$
\frac{\text { Standard Error }(y)=}{\sqrt{\frac{1}{n-2}\left[\sum(y-\bar{y})^{2}-\frac{\sum[(x-\bar{x})(y-\bar{y})]^{2}}{\sum(x-\bar{x})^{2}}\right]}}
$$

- Minimum value: It refers to the least signal point value in a given signal.

- Maximum value: It refers to the extreme signal point value in a given signal.

- Range: Difference in extreme and least signal point values for a given signal.

- Standard deviation: This is a measure of the actual energy or power content of the vibration signal. The following formula was used for calculation of standard deviation.

Standard Deviation $(\sigma)=\sqrt{\frac{n \sum x^{2}-\left(\sum x\right)^{2}}{n(n-1)}}$ (4)

- Sample variance: It is the variance of the signal points and the following formula was used for calculation of sample variance.

$$
\text { Sample Variance }=\frac{n \sum x^{2}-\left(\sum x\right)^{2}}{n(n-1)} \text { (5) }
$$

When the statistical feature extraction was completed, the features were taken and the feature selection method was carried out. The statistical features form the input to the feature selection method. With the selected features, the further classification was carried out.

\section{J48 DECISION TREE ALGORITHM FOR FEATURE SELECTION}

J48 decision tree algorithm is adapted from the $\mathrm{C} 4.5$ algorithm in WEKA [17]. It consists of a number of branches, one root, a number of nodes, and a number of leaves. One branch is a chain of nodes from the root to a leaf, and each node involves one attribute. The occurrence of an attribute in a tree provides information about the importance of the associated attribute [18]. A decision tree is a tree based knowledge representation methodology used to represent classification rules. J48 decision tree algorithm is a widely used one to construct decision trees [19]. The procedure of forming the decision tree and exploiting the same for feature selection is characterized by the following:

1. The set of features available at hand forms the input to the algorithm; the output is the decision tree.

2. The decision tree has leaf nodes, which represent class labels, and other nodes associated with the classes being classified.

3. The branches of the tree represent each possible value of the feature node from which they originate.

4. The decision tree can be used to classify feature vectors by starting at the root of the tree and moving through it until a leaf node, which provides a classification of the instance, is identified.

5. At each decision node in the decision tree, one can select the most useful feature for classification using appropriate estimation criteria. The criterion used to identify the best feature invokes the concepts of entropy reduction and information gain.

Information gain measures how well a given attribute separates the training examples according to their target classification. The measure is used to select the candidate features at each step while growing the tree [20]. Information gain is the expected reduction in entropy caused by portioning the samples according to this feature.

Information gain $(S, A)$ of a feature $A$ relative to a collection of examples $S$, is defined as:

$$
\begin{gathered}
\operatorname{Gain}(S, A)= \\
\operatorname{Entropy}(S)-\sum_{v \in \operatorname{Value}(A)} \times \frac{\left|S_{v}\right|}{|S|} \operatorname{Entropy}\left(S_{v}\right)-(6)
\end{gathered}
$$

where Value $(A)$ is the set of all possible values for attribute $A$, and $S_{v}$ is the subset of $S$ for which feature $A$ has value $v$. Note the first term in the equation for gain is just the entropy of the original collection $S$ and the second term is the expected value of the entropy after $S$ is partitioned using feature $A$. The expected entropy described by the second term is simply the sum of the entropies of each subset $S_{v}$, weighted by the fraction of samples $\left|S_{v}\right| /|\mathrm{S}|$ that belong to $S_{v}$. Gain $(S, A)$ is, therefore, the expected reduction in entropy caused by knowing the value of feature $A$. Entropy is a measure of homogeneity of the set of examples and it is given by

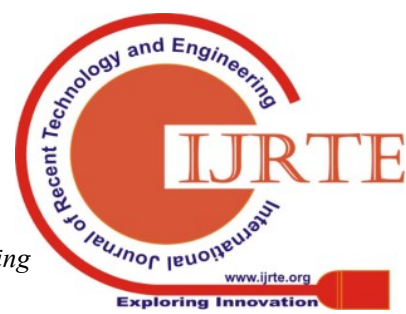




$$
\operatorname{Entropy}(S)=\sum_{i-1}^{c}-P_{i} \log _{2} P_{i}-(7)
$$

where, $c$ is the number of classes, $P_{i}$ is the proportion of $S$ belonging to class' $i$ '.

The J48 decision tree algorithm has been applied to the problem of feature selection. The input to the algorithm is the set of statistical features described above and output of the decision tree shown in Figure 5. It is clearly shown that the top node is the best node for classification. The other features in the nodes of decision tree perform in descending order of significance. It is to be mentioned here that only features that contribute to the classification appear in the decision tree and other features do not contribute much. The features which have the less discriminating capability can be consciously discarded by deciding on the threshold. This concept is made use for selecting good features. Figure 6 shows the classification accuracy for number of features. The algorithm identifies the good features for the purpose of classification of the given training data set, and thus reduces the domain knowledge required to select good features for pattern classification problem [21]. Referring from Figure 5, one can identify the most dominating features to represent the blade conditions are the sum, range, standard deviation, and kurtosis.

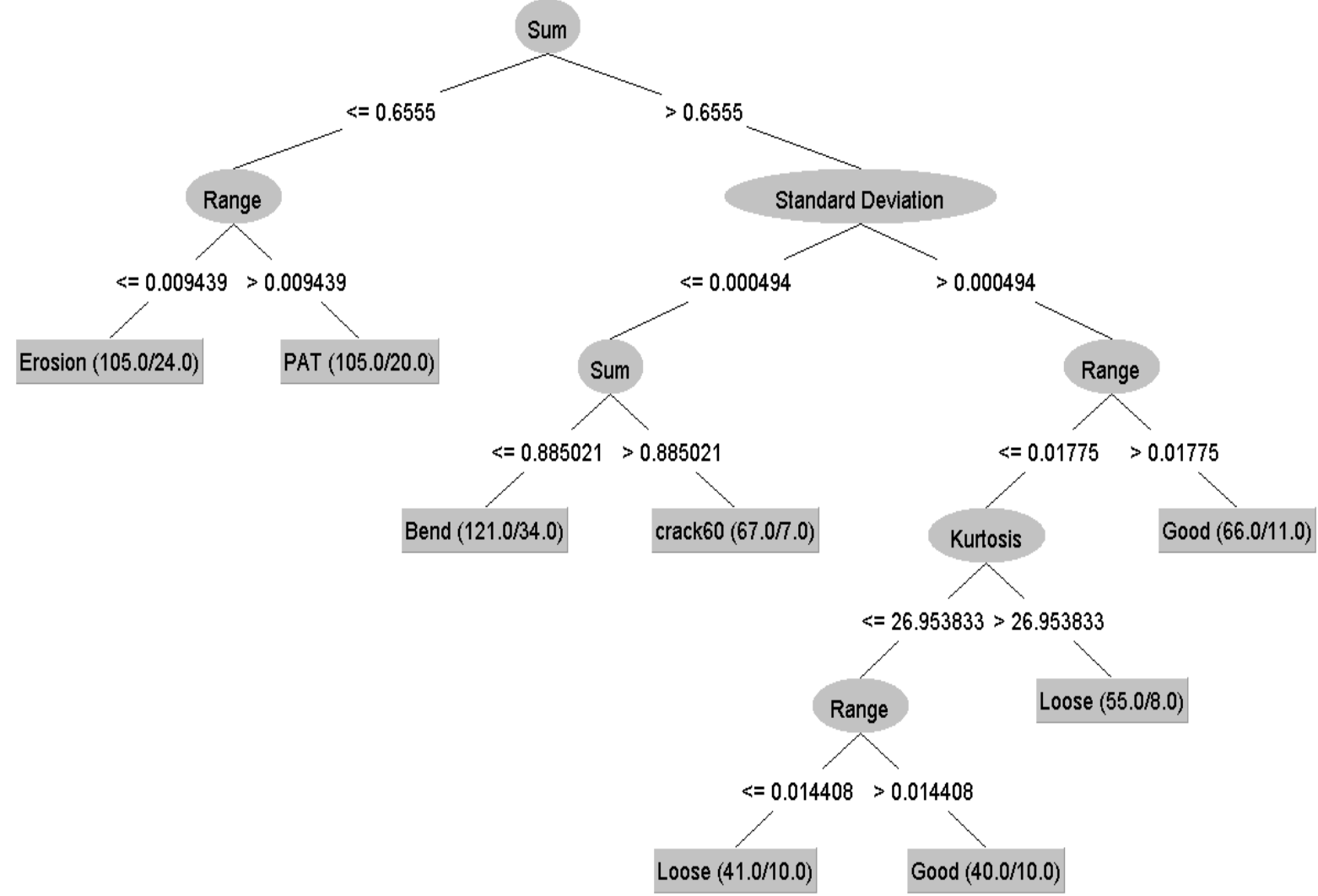

Figure 5: J48 Tree classification for feature selection

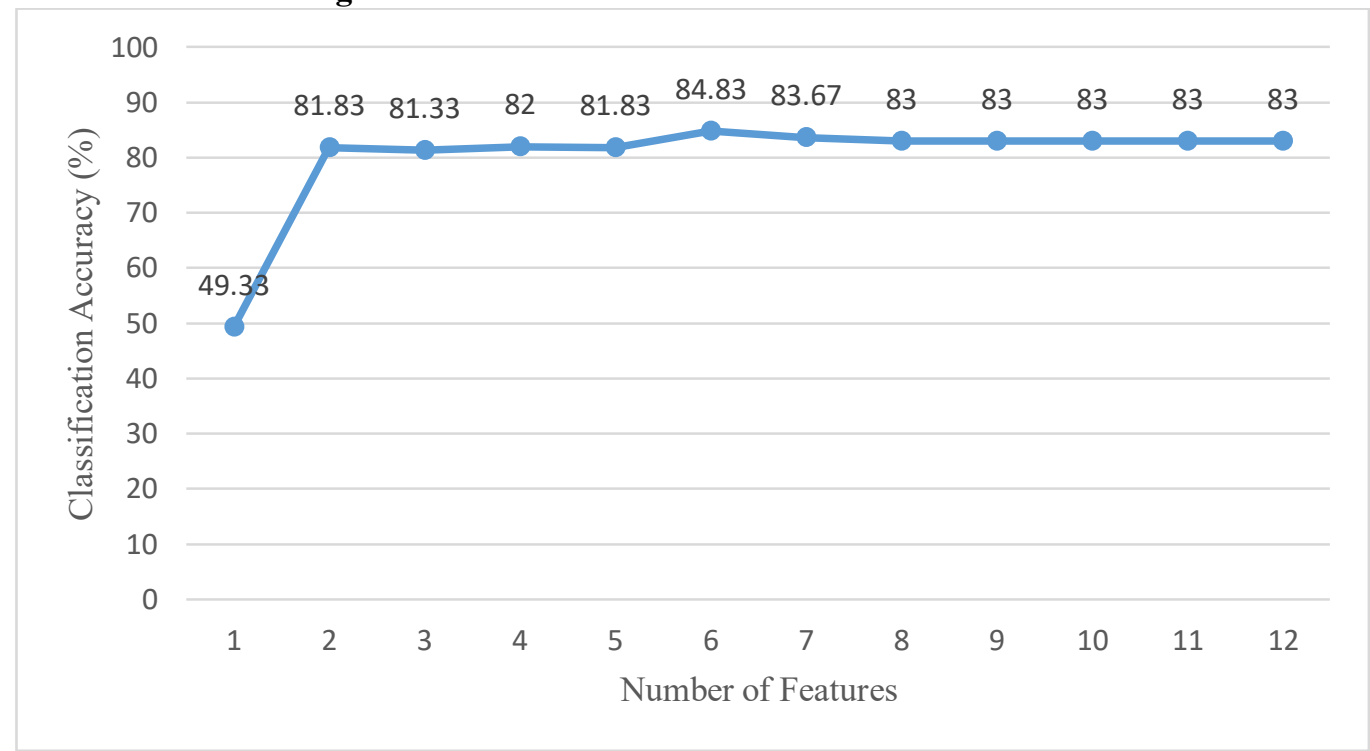

Figure 6: Classification accuracy for number of features 


\section{FEATURE CLASSIFICATION}

After the feature selection, the fault classification was carried out using hyperpipes (HP) and voting feature intervals (VFI) algorithms. These both classifiers are called as miscellaneous Classifiers.

Hyperpipes is an extremely simple classification algorithm that shares the double points of interest of being both quick in operation, and ready to effectively handle huge quantities of features. The standard hyperpipes algorithm can't deal with numeric class information [22].

The essential thought is to make a solitary pipe for every class of the dataset. In training, the channel for every class monitors, which trait values it has experienced, however, no instructions of any sort are kept. At that point, to test every sample is classified by pipe that most contain its abilities, which means the channel that most matches the specific occurrence's sample of abilities [23].

Voting Feature Interval (VFI) is one of the most utilized machine learning approach [24]. The primary qualities of Voting Feature Interval are, it is faster, very precise, powerful too noisy training cases and has short training and classification time. It depends on voting recurrence intervals. In voting Feature Interval, the class name is allotted to every training sample and is communicated as a vector of elements.

The classification of another sample depends on a voting among the classification made by the estimations of every element independently. Attribute intervals are then made for every component from the training illustrations. For every interval, a single value and the votes of every class in that interval are maintained. In this manner, two nearby intervals denote to various classes. The class getting the most elevated vote is pronounced to be the expected class [25].

\section{RESULTS AND DISCUSSION}

The vibration signals were noted for good condition and faulty blade conditions using DAQ. Totally 600 samples were collected; out of which 100 samples were from good condition blade. For different faults such as like blade bends, erosion, blade cracks, hub-blade loose connection, pitch angle twist, 100 samples from every condition were noted.

J48 decision tree algorithm was used to select the best contributing statistical features from twelve features which can have a say in discriminating fault conditions specific sum, mean, median, mode, minimum, maximum, range, skewness, kurtosis, standard error, standard deviation and sample variance.

From Figure 5 and 6, the selected features are given as the input to the classifier to determine the classification accuracy with respect to faults created on the wind turbine blade. From Figure 7, voting feature intervals (VFI) algorithms give the maximum classification accuracy of $75.13 \%$ when compared tohyperpipes (HP) algorithms $(70 \%)$.

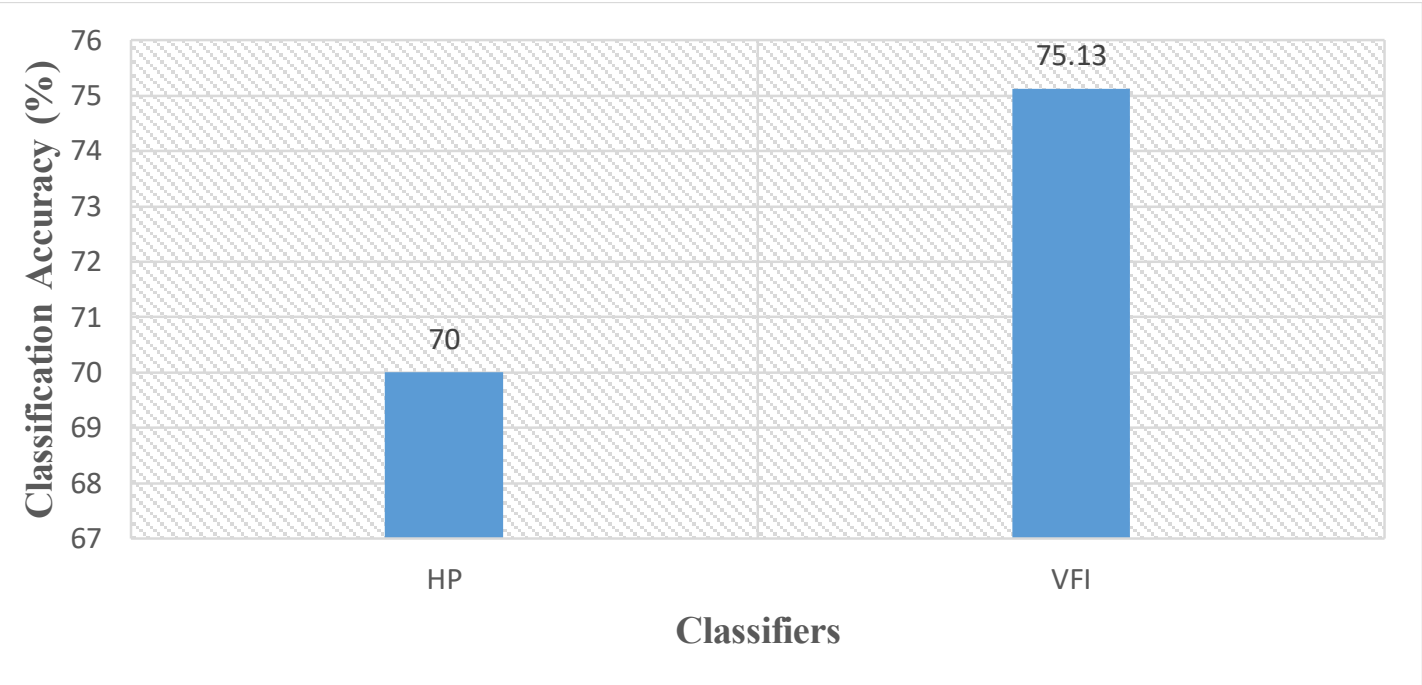

Figure 7: Overall classification accuracy of the classifiers

The strength of bias towards more confident feature the result of bias which is varied for voting feature intervals varied from 0.1 to 20 in voting feature intervals (VFI) algorithms to provide a maximum accuracy with low misclassification errors [26]. For bias 9, the classification accuracy of VFI was found to be $77.67 \%$. Figure 8 shows (VFI) algorithms. The confusion matrix of the voting feature intervals (VFI) algorithms is shown in Table 2. In confusion matrix, the diagonal element represents the correctly classified instance and the others are misclassified [27].

Table 2: Confusion matrix for voting feature intervals (VFI) algorithms

\begin{tabular}{ccccccc}
\hline Blade conditions & Good & Bend & Crack & Loose & PAT & Erosion \\
\hline Good & $\mathbf{8 3}$ & 0 & 1 & 16 & 0 & 0 \\
Bend & 1 & $\mathbf{7 7}$ & 6 & 1 & 0 & 15 \\
Crack & 2 & 8 & $\mathbf{6 1}$ & 3 & 14 & 12 \\
Loose & 20 & 0 & 5 & $\mathbf{6 9}$ & 6 & 0 \\
PAT & 2 & 0 & 0 & 4 & $\mathbf{9 0}$ & 4 \\
Erosion & 1 & 4 & 1 & 0 & 8 & $\mathbf{8 6}$ \\
\hline
\end{tabular}


From 600 samples, 466 samples are correctly classified $(77.67 \%)$ and remaining 134 are misclassified (22.33\%). From confusion matrix (Table 2), the good signal shows 16 faulty signals are classified as loose condition [28]. This does not have much impact when compare with the loose condition being classified as good signals (20 signals). The time taken to build the model is about 0.78 seconds; hence, this can use in real time for the fault detection on the wind turbine blade. The detailed classwise accuracy is shown in Table 3.

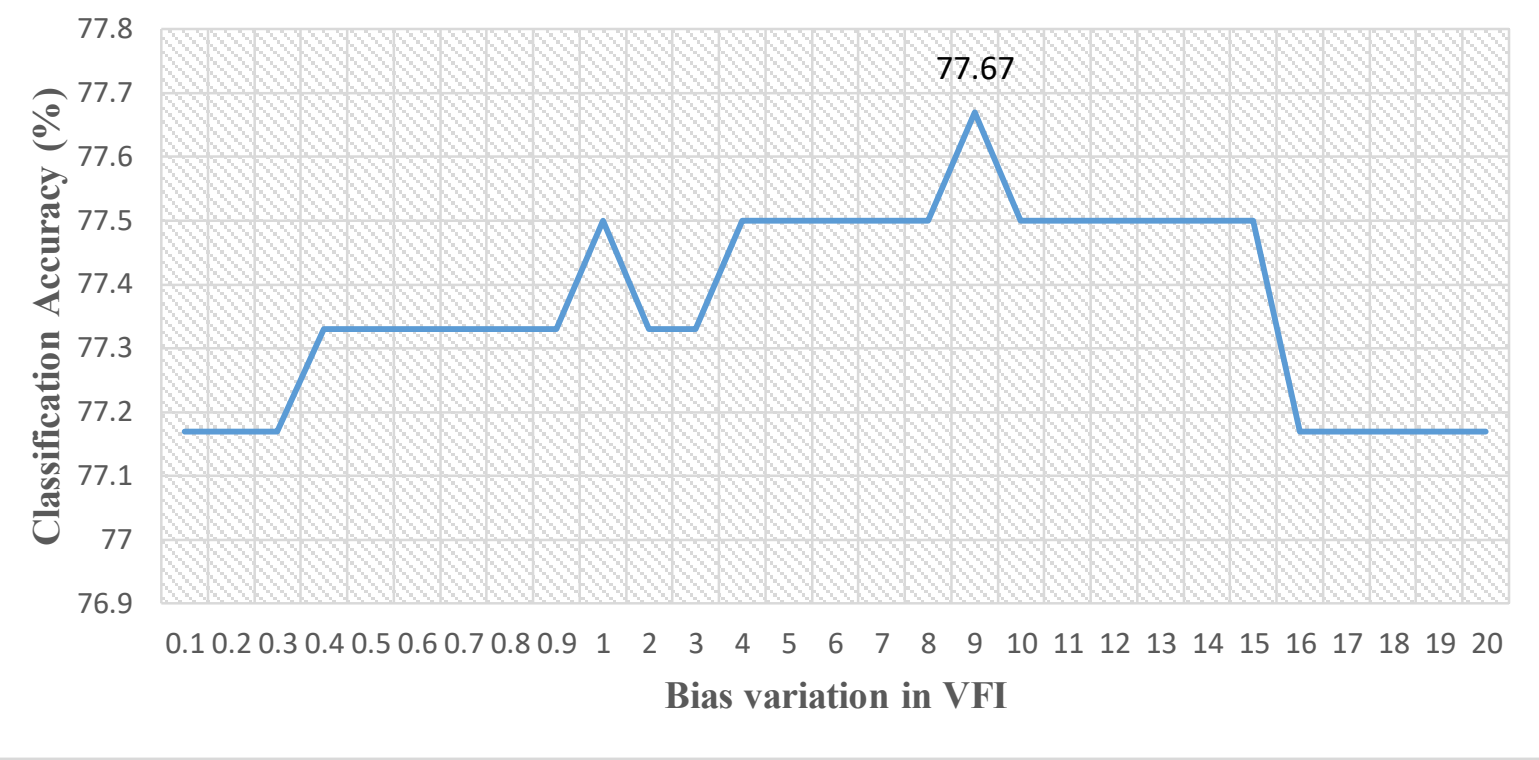

Figure 8: Classification accuracy Vs Bias variation in VFI

Table 3: Classwise accuracy of voting feature intervals (VFI) algorithms

\begin{tabular}{ccccccc}
\hline Class & TP Rate & FP Rate & Precision & Recall & F-Measure & ROC area \\
\hline Good & 0.83 & 0.052 & 0.761 & 0.83 & 0.794 & 0.959 \\
Bend & 0.77 & 0.024 & 0.865 & 0.77 & 0.815 & 0.931 \\
Crack & 0.61 & 0.026 & 0.824 & 0.61 & 0.701 & 0.931 \\
Loose & 0.69 & 0.048 & 0.742 & 0.69 & 0.715 & 0.926 \\
PAT & 0.90 & 0.056 & 0.763 & 0.90 & 0.826 & 0.971 \\
Erosion & 0.86 & 0.062 & 0.735 & 0.86 & 0.793 & 0.949 \\
\hline
\end{tabular}

The kappa statistics were found to be 0.732. It is used to are with the ultimate result. The root mean square error was measure the arrangement of likelihood with the true class.

The mean absolute error was found to be 0.1939 . It is a measure used to measure how close forecasts or prediction

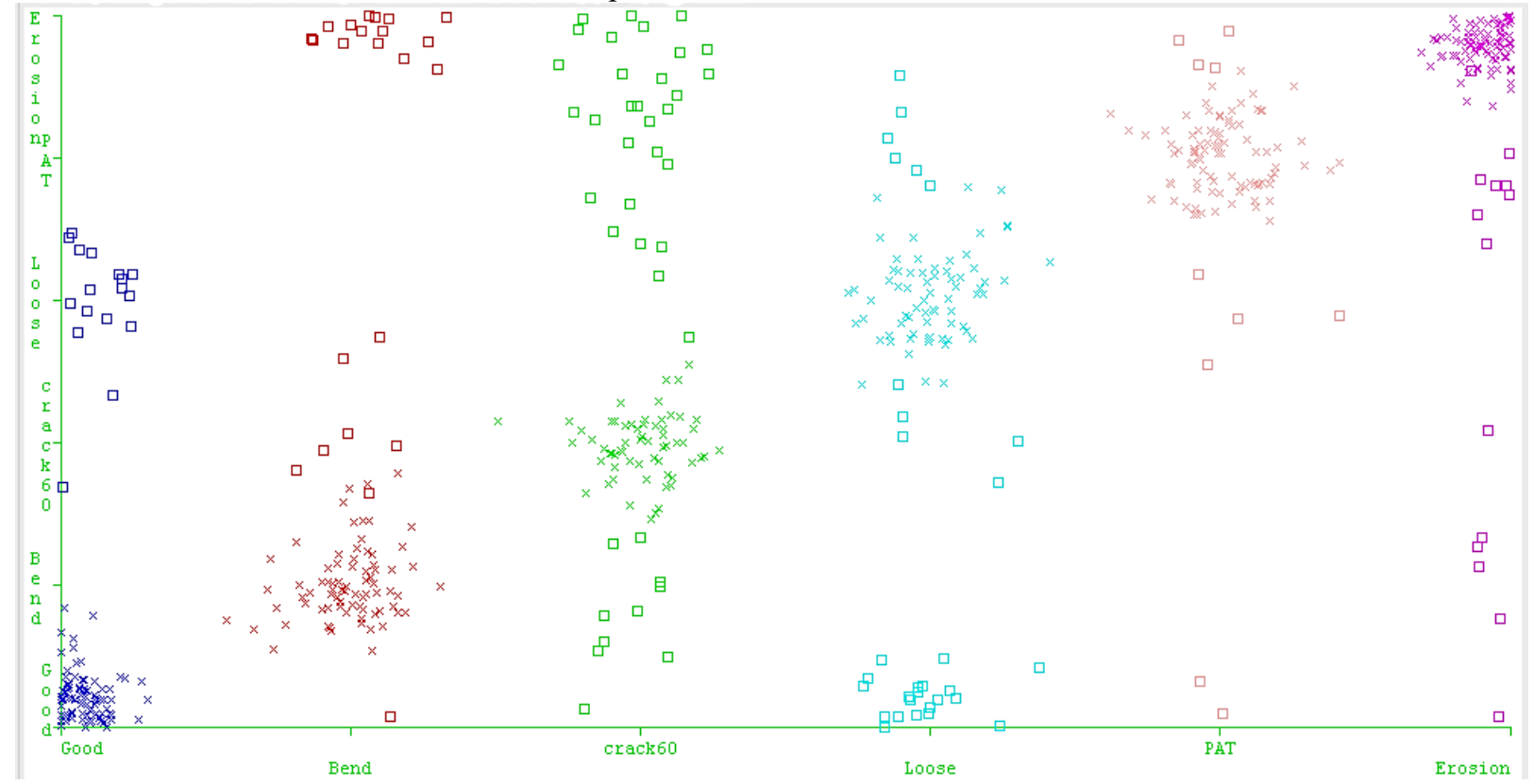

Figure 9: Classifier Errors (Classification vs Misclassification) 
The true positive (TP) rate should be close to 1 and the false positive (FP) rate should be close to 0 to propose the classifier is a better classifier for the problem classification. $\mathrm{TP}$ and FP are very important in the classification process. In the voting feature intervals (VFI) algorithms, it shows that the TP near to 1 and FP close to 0 , then one can predict that the classifier we build for the particular problem is very much effective for the fault diagnosis problem [30]. The classifier error chart is shown in Figure 9. Here the squared dots represent the misclassification and the ' $x$ ' denotes the correct classification.

\section{CONCLUSION}

The wind turbine is very important in the production of wind energy in day to day life. This paper presents an algorithmic based clarification of vibration signals for the assessment of wind turbine blade conditions. From the acquired vibration data, two modelswere developed using data modelling technique. Hyperpipes (HP) and voting feature intervals (VFI) algorithms was used to learn and classify the different conditions of the blade. These models are tested under 10 -fold cross validation and maximum correctly classified instances were found to be $77.67 \%$ for voting feature intervals (VFI) algorithm. The error rate is relatively less and may be considered for the blade fault diagnosis. Hence, voting feature intervals (VFI) algorithm can be practically used for the condition monitoring of wind turbine blade to reduce the downtime and to provide more wind energy. The methodology and algorithm suggested in this paper can be potentially used for any kind of wind turbine blade to diagnose the blade fault with minimal modification.

\section{REFERENCES}

1. Ciang CC, Lee JR, Bang HJ. Structural health monitoring for a wind turbine system: a review of damage detection methods. Measurement Science and Technology. 2008 Oct 13;19(12):122001.

2. Liu WY, Tang BP, Han JG, Lu XN, Hu NN, He ZZ. The structure healthy condition monitoring and fault diagnosis methods in wind turbines: A review. Renewable and Sustainable Energy Reviews. 2015 Apr 30;44:466-72.

3. Tummala A, Velamati RK, Sinha DK, Indraja V, Krishna VH. A review on small scale wind turbines. Renewable and Sustainable Energy Reviews. 2016 Apr 30;56:135171.

4. Joshuva, A., Sugumaran, V. Classification of Various Wind Turbine Blade Faults through Vibration Signals Using Hyperpipes and Voting Feature Intervals Algorithm. International Journal of Performability Engineering. 2017 May;13:247-258.

5. Shokrieh MM, Rafiee R. Simulation of fatigue failure in a full composite wind turbine blade. Composite Structures. 2006 Aug 31;74(3):332-42.

6. Kusiak A, Verma A. A data-driven approach for monitoring blade pitch faults in wind turbines. Sustainable Energy, IEEE Transactions on. 2011 Jan;2(1):87-96.

7. Frost SA, Goebel K, Obrecht L. Integrating Structural Health Management with Contingency Control for Wind Turbines. IJPHM Special Issue on Wind Turbine PHM (Color). 2013:11.

8. Chen B, Matthews PC, Tavner PJ. Wind turbine pitch faults prognosis using a-priori knowledge-based ANFIS
Expert Systems with Applications. 2013 Dec 1;40(17):6863-76.

9. Lee JK, Park JY, Oh KY, Ju SH, Lee JS. Transformation algorithm of wind turbine blade moment signals for blade condition monitoring. Renewable Energy. 2015 Jul 31;79:209-18.

10. Rafiee R, Tahani M, Moradi M. Simulation of aeroelastic behavior in a composite wind turbine blade. Journal of Wind Engineering and Industrial Aerodynamics. 2016 Apr 30;151:60-9.

11. de Bessa IV, Palhares RM, D'Angelo MF, Chaves Filho JE. Data-driven fault detection and isolation scheme for a wind turbine benchmark. Renewable Energy. 2016 Mar 31;87:634-45.

12. Hoell S, Omenzetter P. Optimal selection of autoregressive model coefficients for early damage detectability with an application to wind turbine blades. Mechanical Systems and Signal Processing. 2016 Mar 31;70:557-77.

13. Pourrajabian A, Afshar PA, Ahmadizadeh M, Wood D. Aero-structural design and optimization of a small wind turbine blade. Renewable Energy. 2016 Mar 31;87:83748.

14. Vučina D, Marinić-Kragić I, Milas Z. Numerical models for robust shape optimization of wind turbine blades. Renewable Energy. 2016 Mar 31;87:849-62.

15. Joshuva A, Sugumaran V. Fault diagnostic methods for wind turbine: A review. Asian Research Publishing Network (ARPN) Journal of Engineering and Applied Sciences. 2016 Apr;11(7):4654-4668.

16. Joshuva A, Sugumaran V. A data driven approach for condition monitoring of wind turbine blade using vibration signals through best-first tree algorithm and functional trees algorithm: A comparative study. ISA transactions. 2017 Mar 31; 67:160-72.

17. Joshuva A, Sugumaran V. Wind Turbine Blade Fault Diagnosis Using Vibration Signals through Decision Tree Algorithm. Indian Journal of Science and Technology. 2016 Dec 29;9(48):1-7.

18. Joshuva A, Sugumaran V, Amarnath M. Selecting kernel function of Support Vector Machine for fault diagnosis of roller bearings using sound signals through histogram features. International Journal of Applied Engineering Research. 2015;10(68):482-7.

19. Joshuva A, Sugumaran V. A machine learning approach for condition monitoring of wind turbine blade using autoregressive moving average (ARMA) features through vibration signals: a comparative study. Progress in Industrial Ecology, an International Journal. 2018;12(1-2):14-34.

20. Joshuva A, Sugumaran V. A Comparative Study for Condition Monitoring on Wind Turbine Blade using Vibration Signals through Statistical Features: a Lazy Learning Approach. International Journal of Engineering \& Technology. 2018;7(4.10):190-6.

21. More Vasudha, PanditraoHarshal, Joshuva A, Sugumaran V. Effect of Sampling Frequency and Sample Length on Fault Diagnosis of Wind Turbine Blade. Pakistan Journal of Biotechnology. 2018 ; 15(Special Issue ICRAME 17):14-17.

22. Witten IH, Frank E. Data Mining: Practical machine learning tools and techniques. Morgan Kaufmann; 2005 Jul 13.

23. Deeb ZA, Devine T, Geng Z. Randomized Decimation HyperPipes. Citeseer. 2010.

24. Demiröz G, Güvenir HA. Classification by voting feature intervals. In European Conference on Machine Learning 
1997 Apr 23 (pp. 85-92). Springer Berlin Heidelberg.

25. Ali F, Hayat M. Classification of membrane protein types using Voting Feature Interval in combination with Chou's Pseudo Amino Acid Composition. Journal of theoretical biology. 2015 Nov 7;384:78-83.

26. Joshuva A, Sugumaran V. A Study of Various Blade Fault Conditions on a Wind Turbine Using Vibration Signals through Histogram Features. Journal of Engineering Science and Technology. 2018 Jan;13(1):102-121.

27. Manju BR, Joshuva A, Sugumaran V. A Data Mining Study for Condition Monitoring on Wind Turbine Blades Using Hoeffding Tree Algorithm Through Statistical and Histogram Features. International Journal of Mechanical Engineering and Technology. 2018 ;9(1):1061-1079.

28. Joshuva A, Sugumaran V. A comparative study of Bayes classifiers for blade fault diagnosis in wind turbines through vibration signals. Struct Durab Health Monit (SDHM). 2017;12(1):69-90.

29. Joshuva A, Sugumaran V, Amarnath M, Lee SK. Remaining Life-Time Assessment of Gear Box Using Regression Model. Indian Journal of Science and Technology. 2016 Dec 28;9(47):1-8.

30. Glowacz A. Fault diagnosis of single-phase induction motor based on acoustic signals. Mechanical Systems and Signal Processing. 2019 Feb 15;117:65-80.

31. Deenadayalan, Sivakumar, S., Vishnuvardhan R., Sathish Kumar R, "Fabrication and Characterisation of B-H-G Fiber with Teak Wood Particles Reinforced Hybrid Composite, International Journal of Engineering and Technology, Vol 7(2.31), 208 -211, 2018

32. Sivakumar S, Dhanalakshmi V and Vishuvardhan R "Extraction of Subtractive Features of Prismatic Parts from STEP File for CAD/CAM Integration" Journal of Science and Technology, Malaysia, Vol. 27 (1), 343-356, Jan. 2019.

33. Vishnuvardhan R, Pooranam N, Sivakumar S, Vignesh T., "Producing Electrical Energy from Light Intensity, Design Traking System by Dual Axis Method" International Journal of Mechanical and Production Engineering Research and Development (IJMPERD) Vol. 9, Jan 2019, 503-509. 